\title{
Association between the Estimated Glomerular Filtration Rate and Brain Atrophy in Older Adults
}

\author{
Michael Grasing ${ }^{a} \quad$ Palash Sharma ${ }^{b}$ Rebecca J. Lepping ${ }^{c, d}$ Robyn Honea d, e \\ Jeffrey M. Burns ${ }^{d, e, f} \quad$ William M. Brooks ${ }^{c, d, e, f} \quad$ Aditi Gupta ${ }^{d, e, g, h}$
}

for the Alzheimer's Disease Neuroimaging Initiative

${ }^{a}$ The School of Medicine, University of Kansas Medical Center, Kansas City, KS, USA; ${ }^{b}$ Department of Biostatistics \& Data Science, University of Kansas Medical Center, Kansas City, KS, USA; ${ }^{C}$ Hoglund Biomedical Imaging Center,

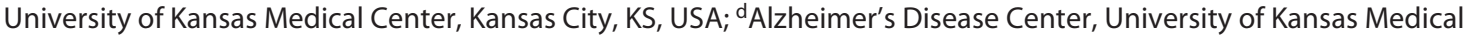
Center, Kansas City, KS, USA; 'Department of Neurology, University of Kansas Medical Center, Kansas City, KS, USA; ${ }^{f}$ Frontiers Clinical \& Translational Science Institute at the University of Kansas, University of Kansas Medical Center, Kansas City, KS, USA; ${ }^{9}$ Department of Internal Medicine, University of Kansas Medical Center, Kansas City, KS, USA; hDivision of Nephrology and Hypertension, University of Kansas Medical Center, Kansas City, KS, USA

\section{Keywords}

Chronic kidney disease - Brain atrophy · Kidney function . Estimated glomerular filtration rate $\cdot$ Cognition

\begin{abstract}
End-stage kidney disease has been associated with cognitive impairment and brain atrophy. It remains unclear if mild to moderate kidney dysfunction is associated with brain atrophy, especially in older adults. We used cross-sectional data from the Alzheimer's Disease Neuroimaging Initiative (ADNI), an $\mathrm{NIH}$-funded multicenter longitudinal cohort study, to better understand the association between estimated glomerular filtration rate (eGFR) and brain volumes. We included all ADNI participants with both baseline serum creatinine values and MRI brain volume assessments. We used multiple linear regression modeling to assess crosssectional associations between eGFR and whole-brain gray matter, hippocampus, entorhinal, fusiform, and middle temporal brain volumes. Participants $(n=1,596)$ were $74 \pm 7$
\end{abstract}

years old with a mean eGFR of $69.4 \pm 14.8 \mathrm{~mL} / \mathrm{min} / 1.73 \mathrm{~m}^{2}$; $53 \%$ had mild cognitive impairment, and $19 \%$ had dementia. Unadjusted analysis showed an association between lower eGFR and smaller brain volumes. After adjusting for age, sex, and education, there was no association between eGFR brain volumes ( $p>0.05$ for all). These results remained consistent after subgroup analysis by age stratification and baseline cognitive status. Age was a confounding variable in the unadjusted association between the eGFR and brain volumes. Thus, a mild to moderately reduced eGFR was not associated with brain atrophy in ADNI participants.

(c) 2022 S. Karger AG, Basel

Data used in preparation of this article were obtained from the Alzheimer's Disease Neuroimaging Initiative (ADNI) database (adni. loni.usc.edu). As such, the investigators within the ADNI contributed to the design and implementation of ADNI and/or provided data but did not participate in analysis or writing of this report. A complete listing of ADNI investigators can be found at: http://adni.loni. usc.edu/wp-content/uploads/how_to_apply/ADNI_Acknowledgement_List.pdf.
Correspondence to:

Aditi Gupta, agupta@kumc.edu 


\section{Introduction}

Cognitive impairment is common in chronic kidney disease (CKD). An association between cognition and $\mathrm{CKD}$ is supported by physiological links between the brain and kidneys; both organs have similar hemodynamic mechanisms and interact through shared signaling mechanisms [1]. Cognitive impairment in CKD is multifactorial and is accompanied by structural and functional changes in the brain, such as brain atrophy, which might be mediated by increased white matter lesions, altered cerebral blood flow, and decreased white matter integrity [2-8]. Although there is mounting evidence for brain atrophy in CKD [4-8], there are studies that did not find an association between kidney function and brain atrophy $[9,10]$. A possible explanation for this discrepancy may be confounding by vascular risk factors and older age commonly associated with CKD. Additionally, in older adults, the clinical significance of mild to moderate lowering of estimated glomerular filtration rate (eGFR) may be different from younger patients, and current equations to calculate eGFR may be less accurate [11].

Cognitive impairment, brain atrophy, and CKD preferentially affect older adults. With increase in life expectancy, assessment of the impact of CKD in the aging population is important. We have previously shown that mild to moderately low eGFR is not associated with lower cognitive function [12] and does not predict cognitive decline [13] in older adults. In this study, we explore the association between mild to moderately low eGFR and brain atrophy using publicly available data from the Alzheimer's Disease Neuroimaging Initiative (ADNI) [14].

\section{Methods}

The ADNI (http://adni.loni.usc.edu/) is a longitudinal observational cohort of participants from across the United States with ages ranging 55-90 years aimed at understanding the progression of dementia. The ADNI includes participants in general good health with normal cognition, mild cognitive impairment (MCI), or dementia. The ADNI excludes those with significant neurological diseases other than possible Alzheimer's disease, such as Parkinson's disease, seizure disorder, subdural hematoma, and multiple sclerosis, significant systemic illness or unstable medical condition, major depression, bipolar disorder, alcohol or substance abuse, or residence in a skilled nursing facility that could interfere with the study. We used data from the three ADNI phases of ADNI (ADNI-1, ADNI-GO, and ADNI-2) but excluded participants from ADNI-3 as they lacked serum creatinine measurements. For this analysis, we included all ADNI participants with both baseline serum creatinine values and brain volume assessments. We obtained baseline demographic information including age, sex, race, ethnicity, years of education, marital status, cognitive status (normal cognition, MCI, or dementia), body mass index, systolic and diastolic blood pressure, history of smoking, and Hachinski Ischemic Score (a clinical tool for differentiating major types of dementia and identifying those with vascular dementia). Kidney function was estimated using the CKD Epidemiology Collaboration (CKD-EPI) equation to calculate the eGFR. We chose the CKD-EPI equation as it is more accurate in older persons and at higher levels of kidney function and is a better predictor of mortality, cardiovascular events, and ESKD when compared to the Modification of Diet in Renal Disease Study equation.

The ADNI neuroimaging standardized procedure has been previously described [15]. Cortical reconstruction and volumetric segmentation of MRI T1-weighted sagittal 3D magnetization-prepared rapid gradient-echo sequences from ADNI 1, ADNI GO, and ADNI 2 were processed by the University of California San Francisco ADNI study team using FreeSurfer version 5.1 image analysis suite [16-18]. We extracted baseline hippocampus, entorhinal cortex, fusiform, middle temporal, whole brain, and intracranial volumes from the ADNIMERGE dataset and normalized raw regional brain volumes by dividing the regional brain volume by the total intracranial volume to account for brain volume differences associated with head size. Further details on ADNI imaging protocols can be found at http://adni.loni.usc.edu/methods/ documents/mri-protocols/.

Baseline characteristics and normalized brain volumes were compared in the different baseline eGFR categories (eGFR $<45$, $45-60,61-90$, and $>90 \mathrm{~mL} / \mathrm{min} / 1.73 \mathrm{~m}^{2}$ ). Unadjusted one-way ANOVA was used to determine differences in continuous variables between the eGFR groups, and a nonparametric Fisher exact test or $\chi^{2}$ test as appropriate was used to determine group differences in frequencies for categorical variables. We ran a linear regression analysis for brain volumetric data using eGFR categories as the independent variable. The reference group was eGFR 61-90 $\mathrm{mL} / \mathrm{min}$. Additionally, we ran separate univariate and multivariate regression analyses with the eGFR as a continuous variable. The primary dependent variables were whole-brain gray matter volume and hippocampal volume (since hippocampal atrophy is a hallmark for dementia). Secondary dependent variables were entorhinal cortex, fusiform, and middle temporal volumes.

Age is a strong predictor for cognitive decline and dementia as well as for a lower eGFR. To minimize confounding age effects and to determine collinearity between the eGFR group and age on their effect on brain volumes, we divided our cohort into tertiles based on age. We further separated the cohort into subgroups based on cognitive status (normal cognition, MCI, and dementia) since dementia is associated with smaller brain volumes. We ran the same regression models for our primary outcomes on these subgroups. Model assumptions were assessed by residual plots and QQ plots and histograms. All statistical analysis and visualization were performed using R-Studio (version 1.3.1073). A $p$ value less than 0.05 was considered statistically significant.

\section{Results}

We included 1,596 ADNI participants with a mean age of $74 \pm 7$ years, 434 with normal cognition, 852 with MCI, and 310 with dementia in the analysis. The proportions 


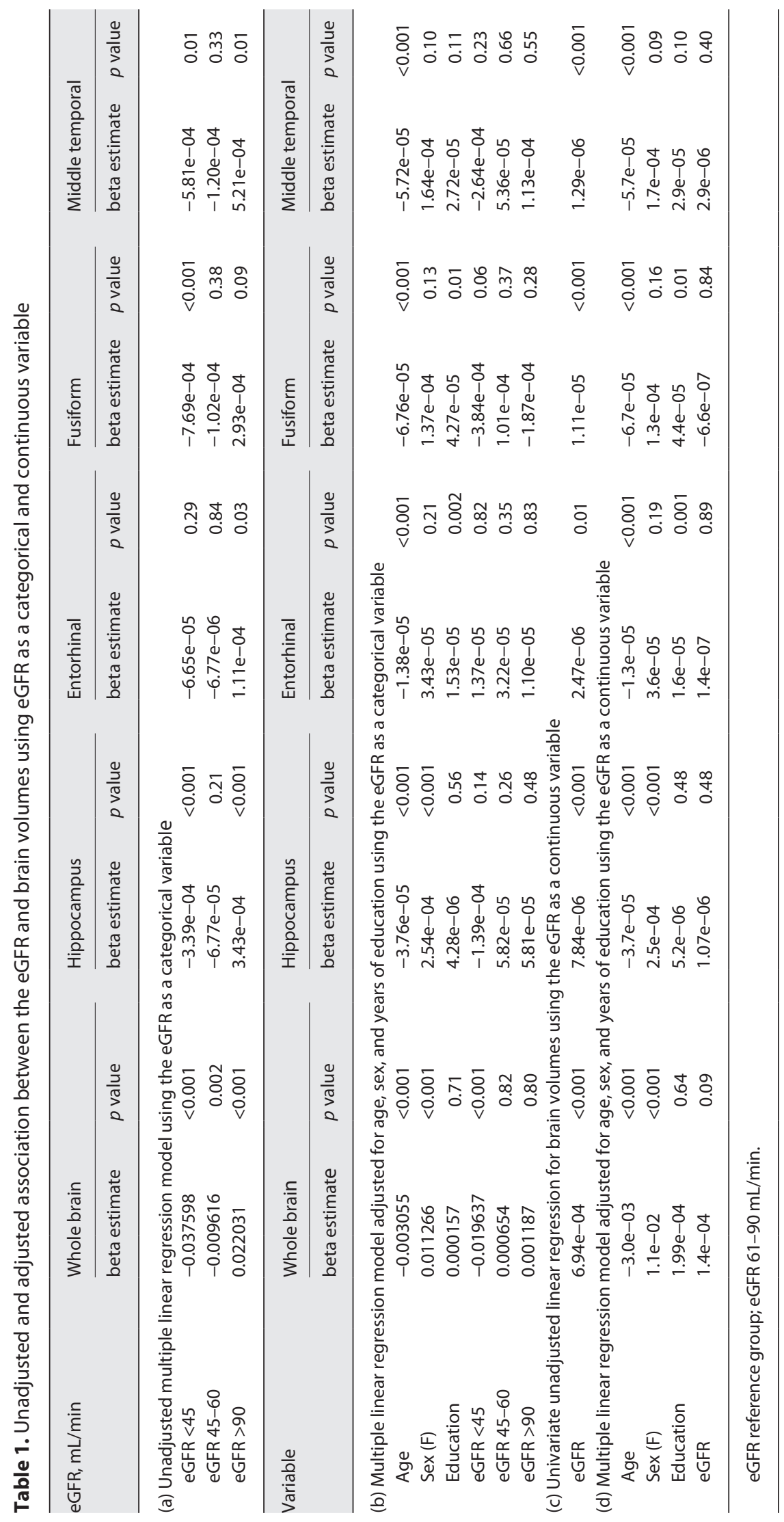


of participants with an eGFR $<45,45-60,61-90$, and $>90$ $\mathrm{mL} / \mathrm{min}$ were $6 \%, 21 \%, 66 \%$, and $7 \%$, respectively. Participants with a higher eGFR were younger (mean ages for an eGFR $<45,45-60,61-90$, and $>90 \mathrm{~mL} / \mathrm{min}$ were $79 \pm$ $7,77 \pm 7,73 \pm 7$, and $67 \pm 6$ years, respectively, $p<0.001)$ and had fewer cardiovascular symptoms $(p<0.001)$, lower Hachinski ischemic scores $(p<0.001)$, and lower prevalence of dementia $(p=0.01)$. Systolic blood pressure did not differ between eGFR groups, but diastolic blood pressure was lower in participants with lower eGFR $(p=0.01)$.

In the unadjusted linear regression model using eGFR categories as an independent variable and the reference group of eGFR $61-90 \mathrm{~mL} / \mathrm{min}$, an eGFR $<45 \mathrm{~mL} / \mathrm{min}$ was associated with smaller normalized brain volumes, except for the entorhinal volume $(p=0.29)$ (Table 1a). Conversely, eGFR $45-60 \mathrm{~mL} / \mathrm{min}$ was not associated with smaller brain volumes, except for the whole-brain volume $(p<0.001)$. eGFR $>90 \mathrm{~mL} / \mathrm{min}$ was associated with larger brain volumes except for the fusiform volume $(p=0.09)$. With adjustment for sex and years of education, eGFR $<45 \mathrm{~mL} / \mathrm{min}$ no longer predicted brain volumes (except lower wholebrain volume for eGFR $<45 \mathrm{~mL} / \mathrm{min}, p<0.001$ ) (Table $1 \mathrm{~b}$ ). Older age was strong predictor for smaller brain volumes. Female sex predicted higher whole-brain and hippocampal volumes, while years of education predicted higher entorhinal and fusiform volumes. Using eGFR as a continuous variable, a lower eGFR was again associated with smaller brain volumes in the univariate analysis (Table 1c). However, after including age, sex, and years of education, in the model, eGFR did not predict any of assessed brain volumes (Table 1d). The only predictor of smaller brain volumes was older age. Female sex predicted higher whole-brain and hippocampal volumes. Years of education predicted higher entorhinal and fusiform volumes.

Participants in age tertile $1-3$ were $66 \pm 4$ years, $74 \pm 2$ years, and $82 \pm 3$ years old, respectively. Participants in tertile 3 were more likely to have dementia, lower eGFR, and body mass index and higher Hachinski ischemic score, systolic blood pressure, and serum creatinine. Normalized brain volumes were smaller in the older age tertiles ( $p<0.001$ for all brain volumes). After separating the cohort by cognitive status (normal cognition, MCI, or dementia) and age tertiles and adjusting for sex and years of education, none of the brain volumes were associated with eGFR in any of the cognition groups. There was a trend $(p=0.08)$ for hippocampus volumes being smaller in participants with normal cognition and a higher eGFR in tertile 3. Sensitivity analysis with participants grouped by eGFR categories showed similar lack of association between the eGFR and brain volumes.

eGFR and Brain Atrophy

\section{Discussion}

These data demonstrate the importance of confounding variables such as age in understanding the association between cognition, brain volumes, and kidney function. Although our initial unadjusted analysis indicated that a lower eGFR was associated with smaller brain volumes, this association was lost after adjusting for confounding variables. These results held true with subgroup analysis based on age and cognition status and with sensitivity analysis using eGFR categories.

It is not surprising that the only predictor of smaller brain volumes was older age in our study as age is a strong risk factor for lower eGFR, brain volumes, cognitive function, and vascular risk factors [19]. Sex also influences brain volumes [20], whereas the association of brain volumes with education is conflicting. After adjusting for age, sex, and years of education, there was no association between the eGFR and brain volumes (except for wholebrain volume and an eGFR $<45 \mathrm{~mL} / \mathrm{min}$ ). The lack of association remained consistent with further sensitivity and subgroup analyses.

Our results are consistent with other studies with a mild to moderately low eGFR $[9,10]$. In the study by Murea et al. [9], only participants with diabetes had a lower intracranial brain volume with a lower eGFR. In fact, in people without diabetes, a lower eGFR was associated with a higher hippocampal gray matter volume. Similarly, Cho et al. [10] studied a large group of individuals with normal cognition and did not find an association between eGFR and cortical thickness. However, only $5 \%$ of their cohort had an eGFR of $<60 \mathrm{~mL} / \mathrm{min}$. Our study adds to these data and confirms that a mild to moderately low eGFR is not associated with low brain volumes. We also had an older cohort compared to the prior studies. In addition, by analyzing data from a relatively healthy cohort, our results are less likely to be confounded. Conversely, other studies associated CKD with smaller brain volumes [4-8]. Some studied younger participants, e.g., in the study by Yakushiji et al. [4], the mean age was 56.4 years. It is possible that a lower eGFR seen with aging may not have the same clinical relevance as a lower eGFR in the young. More studies are needed to understand the possibility of this differential relationship between brain volumes and the eGFR in younger versus older adults.

Due to the cross-sectional nature of our study, definitive conclusions cannot be drawn, and further studies will be needed to establish causality or lack thereof. The ADNI is a convenience sample and lacks diversity; with more than $90 \%$ White participants, the generalizability of our

Am J Nephrol 2022;53:176-181 179 
findings may be limited. Another limitation was the relatively small number of participants with a very low eGFR, such that our results may not be applicable to patients with severe CKD. As is common in CKD studies, we used estimated and not measured GFR to access kidney function since this was available in the ADNI dataset. Although the CKD-EPI equation is more accurate in older adults compared to the Modification of Diet in Renal Disease Study equation, it can overestimate GFR in those with sarcopenia (as sarcopenia is more prevalent with older age and dementia). Also, since the ADNI was not designed to assess the effect of kidney function on cognition, other important markers of kidney function such as albuminuria were not available. Cho et al. [10] previously found albuminuria to be predictive of cortical thickness, whereas the eGFR was not.

In conclusion, we did not find an association between a mild to moderately low eGFR and brain atrophy in the ADNI cohort of older adults. Apparent associations between eGFR and brain volumes may be confounded by aging and other vascular risk factors commonly seen in CKD.

\section{Acknowledgements}

Data collection and sharing for this project was funded by the ADNI (National Institutes of Health Grant U01 AG024904) and Department of Defense (DOD) ADNI (award number W81XWH-12-2-0012). ADNI is funded by the National Institute on Aging and the National Institute of Biomedical Imaging and Bioengineering and through generous contributions from the following: AbbVie, Alzheimer's Association; Alzheimer's Drug Discovery Foundation; Araclon Biotech; BioClinica, Inc.; Biogen; BristolMyers Squibb Co.; CereSpir, Inc.; Cogstate; Eisai, Inc.; Elan Pharmaceuticals, Inc.; Eli Lilly and Company; Euroimmun; F. Hoffmann-La Roche Ltd. and its affiliated company Genentech, Inc.; Fujirebio; GE Healthcare; IXICO Ltd.; Janssen Alzheimer Immunotherapy Research \& Development, LLC.; Johnson \& Johnson Pharmaceutical Research \& Development, LLC.; Lumosity; Lundbeck; Merck \& Co., Inc.; Meso Scale Diagnostics, LLC.; NeuroRx Research; Neurotrack Technologies; Novartis Pharmaceuticals Corporation; Pfizer Inc.; Piramal Imaging; Servier; Takeda Pharmaceutical Co.; and Transition Therapeutics. The Canadian Institutes of Health Research is providing funds to support ADNI clinical sites in Canada. Private sector contributions are facilitated by the Foundation for the National Institutes of Health (www.fnih. org). The grantee organization is the Northern California Institute for Research and Education, and the study is coordinated by the Alzheimer's Therapeutic Research Institute at the University of Southern California. ADNI data are disseminated by the Laboratory for NeuroImaging at the University of Southern California.

\section{Statement of Ethics}

Informed consent was obtained from the ADNI participants at the individual participating sites. The protocol was submitted to the appropriate Institutional Review Boards, and written approval was obtained.

\section{Conflict of Interest Statement}

The authors have no conflicts of interest to declare.

\section{Funding Sources}

This work is supported by the National Institutes of Health (K23 AG055666) to AG Cognitive Impairment in End Stage Renal Disease and (P30 AG035982) to KU ADC and the National Institutes of Health Clinical and Translational Science Awards Grant (UL1 TR000001) to KUMC.

\section{Author Contributions}

Michael Grasing: drafting the manuscript and interpretation of data. Palash Sharma: statistical analysis and interpretation of data. Rebecca J. Lepping: study concept and design, preparation of manuscript, interpretation of data, and editing manuscript. Robyn Honea: study concept and design, interpretation of data, and editing manuscript. Jeffrey M. Burns: critical review and editing manuscript. William M. Brooks: critical review, interpretation of data, and editing manuscript. Aditi Gupta: study concept and design, interpretation of data, and drafting and revision of the manuscript.

\section{Data Availability Statement}

The data that support the findings of this study are openly available in the ADNI database at http://adni.loni.usc.edu/.

\section{References}

1 Afsar B, Sag AA, Yalcin CE, Kaya E, Siriopol $\mathrm{D}$, Goldsmith $\mathrm{D}$, et al. Brain-kidney crosstalk: definition and emerging evidence. Eur J Intern Med. 2016;36:7-12

2 Gupta A, Lepping RJ, Yu AS, Perea RD, Honea RA, Johnson DK, et al. Cognitive function and white matter changes associated with renal transplantation. Am J Nephrol. 2016;43(1):50-7.
3 Lepping RJ, Montgomery RN, Sharma P, Mahnken JD, Vidoni ED, Choi I-Y, et al. Normalization of cerebral blood flow, neurochemicals, and white matter integrity after kidney transplantation. J Am Soc Nephrol. 2021;32(1):177. 
4 Yakushiji Y, Nanri Y, Hirotsu T, Nishihara M, Hara M, Nakajima J, et al. Marked cerebral atrophy is correlated with kidney dysfunction in nondisabled adults. Hypertens Res. 2010; 33(12):1232-7.

5 Tsuruya K, Yoshida H, Haruyama N, Fujisaki K, Hirakata H, Kitazono T. Clinical significance of fronto-temporal gray matter atrophy in executive dysfunction in patients with chronic kidney disease: the VCOHP Study. PLoS One. 2015;10(12):e0143706.

6 Chang CY, Lin CC, Tsai CF, Yang WC, Wang $\mathrm{SJ}$, Lin FH, et al. Cognitive impairment and hippocampal atrophy in chronic kidney disease. Acta Neurol Scand. 2017;136(5):477-85.

7 Yeh Y-C, Kuo Y-T, Huang M-F, Hwang S-J, Tsai J-C, Kuo M-C, et al. Association of brain white matter lesions and atrophy with cognitive function in chronic kidney disease. Int $J$ Geriatr Psychiatry. 2019;34(12):1826-32.

8 Tsuruya K, Yoshida H, Kuroki Y, Nagata M, Mizumasa T, Mitsuiki K, et al. Brain atrophy in peritoneal dialysis and CKD stages 3-5: a cross-sectional and longitudinal study. Am J Kidney Dis. 2015;65(2):312-21.

9 Murea M, Hsu FC, Cox AJ, Hugenschmidt $\mathrm{CE}, \mathrm{Xu}$ J, Adams JN, et al. Structural and functional assessment of the brain in European
Americans with mild-to-moderate kidney disease: Diabetes Heart Study - MIND. Nephrol Dial Transplant. 2015;30(8):1322-9.

10 Cho EB, Shin HY, Park SE, Chun P, Jang HR, Yang JJ, et al. Albuminuria, cerebrovascular disease and cortical atrophy: among cognitively normal elderly individuals. Sci Rep. 2016;6:20692.

11 Ellam T, Twohig H, Khwaja A. Chronic kidney disease in elderly people: disease or disease label? BMJ. 2016;352:h6559.

12 Gupta A, Kennedy K, Perales-Puchalt J, Drew D, Beddhu S, Sarnak M, et al. Mild-moderate $\mathrm{CKD}$ is not associated with cognitive impairment in older adults in the Alzheimer's disease neuroimaging initiative cohort. PLoS One. 2020;15(10):e0239871.

13 Grasing M, Kennedy K, Sarnak MJ, Burns JM, Gupta A. Mild to moderate decrease in eGFR and cognitive decline in older adults. Nephrol Dial Transplant. 2021. Epub ahead of print.

14 Glassock RJ, Rule AD. The implications of anatomical and functional changes of the aging kidney: with an emphasis on the glomeruli. Kidney Int. 2012;82(3):270-7.

15 Jack CR Jr, Bernstein MA, Fox NC, Thompson P, Alexander G, Harvey D, et al. The Al- zheimer's disease neuroimaging initiative (ADNI): MRI methods. J Magn Reson Imaging. 2008;27(4):685-91.

16 Fischl B, Liu A, Dale AM. Automated manifold surgery: constructing geometrically accurate and topologically correct models of the human cerebral cortex. IEEE Trans Med Imaging. 2001;20(1):70-80.

17 Fischl B, Salat DH, Busa E, Albert M, Dieterich $\mathrm{M}$, Haselgrove $\mathrm{C}$, et al. Whole brain segmentation: automated labeling of neuroanatomical structures in the human brain. Neuron. 2002;33(3):341-55.

18 Han X, Jovicich J, Salat D, van der Kouwe A, Quinn B, Czanner S, et al. Reliability of MRIderived measurements of human cerebral cortical thickness: the effects of field strength, scanner upgrade and manufacturer. Neuroimage. 2006;32(1):180-94.

19 Gupta A, Burns JM. A single point-in-time eGFR is not associated with increased risk of dementia in the elderly. J Am Soc Nephrol. 2020;31(12):2965.

20 Coffey CE, Lucke JF, Saxton JA, Ratcliff G, Unitas LJ, Billig B, et al. Sex differences in brain aging: a quantitative magnetic resonance imaging study. Arch Neurol. 1998; 55(2):169-79. 